\title{
ACEITES ESENCIALES PROVENIENTES DE PLANTAS NATIVAS PARA EL CONTROL DE HONGOS FITOPATÓGENOS QUE AFECTAN A FRUTALES
}

\author{
Stegmayer, M. I. ${ }^{1}$; FernándeZ, L. N. ${ }^{1,2} ;$ ÁlvareZ, N. H. ${ }^{1,3}$ \\ Olivella, L. ${ }^{1} ;$ Gutiérrez, H. F.4; Favaro, M. A. ${ }^{1,2}$ \& Derita, M. G. ${ }^{1,5}$
}

\begin{abstract}
RESUMEN
El empleo constante y excesivo de fungicidas sintéticos en la industria fruti-hortícola ha provocado profundas alteraciones en el medio ambiente y en la salud humana. En este contexto, explorar fuentes naturales antifúngicas resulta de importancia económica y medioambiental. Se propuso estudiar la actividad de cinco aceites volátiles obtenidos de las especies vegetales nativas Dysphania ambrosioides, Baccharis frenguellii, Baccharis salicifolia, Lippia alba y Lippia turbinata contra hongos fitopatógenos de los géneros Botrytis, Colletotrichum, Rhizopus, Fusarium y Monilinia, aislados de frutillas y duraznos con sintomatología. La evaluación se realizó mediante el método de difusión en agar adaptado para muestras volátiles y se determinaron los porcentajes de inhibición fúngica para cada muestra en estudio utilizando el software Image $\mathrm{B}$. Se analizó el perfil químico de cada aceite por CG-EM y se determinaron los compuestos mayoritarios. Los resultados indicaron un alto potencial antifúngico de todas las muestras evaluadas, particularmente el producto obtenido de L. alba.
\end{abstract}

Palabras clave: fungicidas, aceites esenciales, plantas nativas, frutas.

1.- ICiAgro Litoral, Universidad Nacional del Litoral-CONICET, Facultad de Ciencias Agrarias. Kreder 2805. 3080HOF, Esperanza, Santa Fe, Argentina.

2.- Cátedra de Fitopatología, Facultad de Ciencias Agrarias, Universidad Nacional del Litoral. Kreder 2805. 3080HOF, Esperanza, Santa Fe, Argentina.

3.- Cátedra de Cultivos Intensivos, Facultad de Ciencias Agrarias, Universidad Nacional del Litoral. Kreder 2805. 3080HOF, Esperanza, Santa Fe, Argentina.

4.- Cátedra de Diversidad de Plantas II, Facultad de Humanidades y Ciencias, Universidad Nacional del Litoral. Ciudad Universitaria-Paraje el Pozo. 3000, Santa Fe, Argentina.

5.- Cátedra de Farmacognosia, Facultad de Ciencias Bioquímicas y Farmacéuticas, Universidad Nacional de Rosario. Suipacha 531, S2002LRK, Rosario, Argentina. Email: mderita@fca.unl.edu.ar, mgderita@hotmail.com Manuscrito recibido el 16 de abril de 2020 y aceptado para su publicación el 2 de junio de 2020 .

Stegmayer, M. I.; Fernández, L. N.; Álvarez, N. H.; Olivella, L.; Gutiérrez, H. F.; Favaro, M. A.; Derita, M. G. Aceites esenciales provenientes de plantas nativas para el control de hongos fitopatógenos que afectan a frutales. FAVE - Ciencias Agrarias 20 (1): 317-329. CC BY-NC-SA 4.0 


\begin{abstract}
Essential oils from native plants for the control of phytopathogenic fungi that affect fruits.

The constant and excessive use of synthetic fungicides in fruit and vegetable industry has caused dangerous environmentally changes and deeply affected human health. In this context, exploring natural antifungal sources represents an economic and environmental importance. It was proposed to study the activity of five volatile oils obtained from the native plant species Dysphania ambrosioides, Baccharis frenguellii, Baccharis salicifolia, Lippia alba and Lippia turbinata against phytopathogenic fungi belonging to the genus Botrytis, Colletotrichum, Rhizopus, Fusarium and Monilinia isolated from infected strawberries and peaches. The assays were carried out using the agar diffusion method adapted for volatile samples and the percentages of fungal inhibition were determined for each sample using Image $®$ software. The chemical profile of each essential oil was analyzed by GC-MS and the majority compounds were determined. Results showed a high antifungal potential for all the samples evaluated, particularly the product obtained from L. alba.
\end{abstract}

Key words: fungicides, essential oils, native plants, fruits.

\section{INTRODUCCIÓN}

Las enfermedades de frutas y hortalizas causadas por hongos fitopatógenos son responsables de grandes pérdidas económicas asociadas principalmente a la reducción del rendimiento de los cultivos, pero también a la disminución de la calidad y seguridad alimentaria del producto. El uso indiscriminado y excesivo de distintos agentes fungicidas, representa un riesgo para la salud humana y animal debido a la contaminación de los alimentos y a la acumulación de residuos tóxicos en el medio ambiente. Como consecuencia de la globalización de los mercados y el cambio climático, este problema está creciendo a un ritmo acelerado (Pergomet et al., 2018), por lo que existe un consenso generalizado en ámbitos académicos y productivos que intenta buscar otras soluciones ambientalmente ami- gables. Los productos naturales de plantas ofrecen un conjunto de agentes antifúngicos estructuralmente diferentes que podrían representar una alternativa a los fungicidas sintéticos para el control de hongos fitopatógenos (Singh et al., 2003). Estos compuestos son selectivamente activos frente a distintas especies fúngicas, biodegradables y potencialmente adecuados para ser usados como agroquímicos en programas integrados de control de enfermedades (Kim et al., 2004). Investigar y experimentar nuevas técnicas que mejoren la calidad de las frutas frescas para el mercado interno o para la industria, y especialmente las destinadas a exportaciones, es una prioridad fundamental para el desarrollo frutícola de nuestro país, sobre todo si contribuyen a mantener muy bajo el grado de contaminación ambiental (Di Liberto et al., 2019). 


\section{Antecedentes de las especies vegetales a evaluar}

Dysphania ambrosioides (L.) Mosyakin \& Clemants (Amaranthaceae): N.V: Paico. Es una hierba perenne y aromática originaria de América pero naturalizada en regiones templadas de casi todo el mundo. En Argentina habita desde Río Negro hacia el norte y es frecuente en suelos modificados, fértiles y húmedos (Alonso y Desmarchelier, 2005). A partir de sus hojas y frutos se preparan infusiones o cocimientos con fines digestivos, antihelmínticos, estimulantes y sudoríficos (Eyssartie et al., 2009; Navone et al., 2014). En ensayos in-vitro, el aceite esencial demostró actividad fungicida frente a Trichophyton rubrum, Microsporum gypseum y otros dermatofitos de importancia clínica en humanos (Prasad et al., 2010). Además, se demostró su actividad inhibitoria contra los fitopatógenos Aspergillus flavus, A. glaucus, A. niger, Colletotrichum gloesporioides, C. musae y Fusarium oxysporum (Jardim et al., 2008).

Baccharis frenguellii Cabrera (Asteraceae): N.V. Chilca. Son arbustos de hasta 1,5 $\mathrm{m}$ de altura distribuidos desde el sur de Brasil, Paraguay y nordeste de la Argentina, hasta Entre Ríos y sur de Santa Fe. Si bien esta especie no presenta usos reportados, las especies del género Baccharis conocidas como carquejas, se utilizan como tónicas, digestivas, diuréticas, antidiarreicas, febrífugas y antihelmínticas. Su cocimiento se aplica en lavajes para casos de reuma, llagas venéreas, heridas y quemaduras (Martínez et al., 2005). Respecto a antecedentes de actividad antifúngica, Feresín et al. (2001) demostraron que el extracto hexánico y diclorometano de otra especie de Baccharis (B. grisebachii) posee un amplio espectro de acción inhibiendo los siguien- tes patógenos humanos a concentraciones menores de $500 \mu \mathrm{g} / \mathrm{mL}$ : Cryptococcus neoformans, Microsporum canis, M. gypseum, Trichophyton mentagrophytes, T. rubrum y Epidermophyton floccosum (Feresín et al., 2001).

Baccharis salicifolia (Ruiz y Pav.) Pers. (Asteraceae): N.V. Chilca amarga. Son arbustos de hasta $2 \mathrm{~m}$ de alto, ramosos, revestidos por nudos pilosos y ampliamente distribuidos desde el sur de los Estados Unidos hasta Chile y Argentina, donde crece en casi todas las provincias. La infusión de sus hojas y ramas frondosas se aplica externamente como un remedio para la inflamación (Martínez et al., 2005). La decocción de sus gajos es utilizada como antitumoral, anti-reumática y anti-sifilítica (Freire et al., 2007). Entre los principales componentes de su aceite esencial figuran: $\alpha$-tuyona, $\alpha$-pineno, sabineno, mirceno y limoneno (Carrizo Flores et al., 2009). Estos autores demostraron también su actividad antibacteriana contra bacterias Gram positivas y Gram negativas a concentraciones inhibitorias entre 0,47 a $0,94 \mu \mathrm{g} / \mathrm{ml}$ (Carrizo Flores et al., 2009). Por otra parte, Ávila-Sosa et al. (2011) utilizaron extractos clorofórmicos de $B$. salicifolia contra el fitopatógeno C. gloeosporioides inhibiendo su crecimiento en un 88\% (Ávila-Sosa et al., 2011).

\section{Lippia alba (Mill.) N.E.Br. ex Britton} \& P.Wilson (Verbenaceae): N.V. Salvia morada. Son arbustos de hasta $1,5 \mathrm{~m}$ de alto, aromáticos y con ramas delgadas, ampliamente distribuidos en Sudamérica tropical y subtropical. La decocción de sus hojas frescas se usa como analgésico, eupéptico y para tratar trastornos gastrointestinales como espasmos e indigestión (Di Stasi et 


\section{I. Stegmayer et al.}

al., 1994). En Chaco se ha descripto que esta especie también se usa como antitusiva, descongestiva, para el dolor de garganta y dolores de cabeza como también de forma externa para tratar enfermedades cutáneas, quemaduras, heridas y úlceras (Scarpa, 2004). El rendimiento del aceite esencial obtenido de sus hojas es variable (entre 0,5 y $1,5 \%$ ), siendo su componente principal el limoneno junto con p-cimeno, $\alpha$-pineno, alcanfor, linalol y timol (Terblanché y Korneliu, 1996) y dependiendo del perfil químico se han definido varios quimiotipos (Oliveira et al., 2006). El aceite esencial de L. alba mostró actividades inhibitorias contra hongos aflatoxigénicos como A. flavus, siendo sus concentraciones inhibitorias más bajas que las del fungicida sistémico a base de carbendazim Bavistin ${ }^{\circledR}$ (Shukla et al., 2009). Además, fue evaluado en la germinación de teleutosporas de Ustilago scitaminea y de conidios de Colletotrichum falcatum y Curvularia lunata (Rao et al., 2000), siendo todos inhibidos con concentraciones desde 1000 a 4000 ppm. Otros hongos fitopatógenos como Fusarium sp., Penicillium funiculosum y Sclerotinia sclerotiorum también fueron inhibidos a concentraciones de 1000 $\mu \mathrm{g} / \mathrm{mL}$ (de Oliveira et al., 2019).

Lippia turbinata Griseb. (Verbenaceae): N.V. Poleo. Son arbustos aromáticos de 1 a $2 \mathrm{~m}$ de altura, muy ramificados y cuya distribución se extiende por el centro y oeste de la Argentina, habitando principalmente en las provincias fitogeográficas del Chaco, Monte y del Espinal. La parte aérea se emplea como digestivo, diurético, tónico, emenagogo, abortivo y para curar el "empacho" (Campos-Navarro y Scarpa, 2013). Sus hojas presentan compuestos de tipo iridoides, flavonoides y su aceite esencial está compuesto principalmente por lipiona (1,2-epoxipulegona), limoneno, cineol, lipiafenol, dihidrolipiona y carvona, entre otros (Terblanche y Kornelius, 1996). Extractos metanólicos de plantas recolectadas en la zona de Tucumán exhibieron actividad antimicrobiana contra bacterias Gram positivas y Gram negativas a dosis equivalentes al antibiótico cloranfenicol (Hernández et al., 2000). La exposición de maníes a vapores del aceite esencial de poleo permitió evitar la contaminación con hongos del género Aspergillus (Passone y Etchevery, 2014).

\section{Antecedentes de los hongos fitopatógenos a controlar}

Botrytis cinerea Pers.: causa los «mohos grises» o las «pudriciones blandas por moho gris» de frutos y hortalizas, afecta desde el estado de plántula hasta la pos-cosecha incluyendo tizón de las inflorescencias y podredumbres de diferentes órganos. Para nuestra región, su amenaza principal se manifiesta en los cultivos de frutillas, siendo el agente responsable de grandes pérdidas económicas (You et al., 2016). Colletotrichum sp: la antracnosis y podredumbre de corona causada por un complejo de especies fúngicas del género Colletotrichum son enfermedades de gran impacto en frutilla pudiendo afectar hojas, corona y fruta, provocando grandes pérdidas productivas. Las especies más frecuentemente asociadas a frutilla son: C. acutatum, C. gloesporoides y $C$. fragariae. Todas producen síntomas semejantes, pero la podredumbre de frutos está asociada con mayor frecuencia a C. acutatum. La infección por este complejo puede permanecer latente, asintomática, y por ello es difícil de detectar (González, 2017). No existen cultivares de frutillas re- 
sistentes a la antracnosis y debido a que las condiciones predisponentes de la enfermedad son temperaturas entre 15 y $30{ }^{\circ} \mathrm{C}$ y humedad ambiente elevada, aparece frecuentemente en las frutas cerca de la cosecha, cuando los productos químicos para controlarlo son escasos debido a los tiempos de carencia que deben respetarse. Por ello, las medidas son preventivas y en Argentina los productos para su tratamiento son Benomil y la mezcla Azoxistrobina + Difeconazole (CASAFE, 2017). Actualmente no hay fungicidas orgánicos aceptables que sean efectivos contra la antracnosis (Bolda et al., 2017). Rhizopus stolonifer (Ehrenb.: Fr.) Vuill: la pudrición blanda de frutos y hortalizas ocasionada por hongos del género Rhizopus se encuentra ampliamente distribuida en todo el mundo y aparece en órganos carnosos de hortalizas, en plantas florales y en frutos que han sido cosechados. Al ser una típica enfermedad de pos-cosecha, sólo adquiere relevancia durante el almacenamiento, transporte y venta en el mercado de estos productos (Salem et al., 2016). Fusarium semitectum Berk. \& Ravenel: Es un patógeno habitual en países tropicales y subtropicales, siendo comúnmente reconocido como un parásito débil. Es responsable de podredumbres de frutos, especialmente en pos-cosecha, aunque en Argentina existen registros de éste patógeno causando cancros en frutos de Nuez (Seta et al., 2004). En el año 2015, Munitz reporta por primera vez en Tucumán la presencia de $F$ semitectum en arándanos que fueron evaluados inmediatamente luego de la cosecha (Munitz, 2015). Además determinó que éste patógeno tiene la capacidad de producir, en baja concentración, micotoxinas (zearalenonas), información que lo hace un patógeno de importancia relevante en frutas. Monilinia fructicola (Winter) Honey: es el agente causal de la enfermedad conocida como tizón de la flor o pudrición parda/marrón/ morena de los frutos con carozo. Es la enfermedad fúngica más importante que afecta al cultivo de durazneros en América del Sur. Es de muy difícil manejo cuando existen condiciones favorables para su desarrollo (temperatura y humedad), debido a la cantidad de aplicaciones de fungicidas que son necesarias para proteger al fruto a lo largo de su amplio período de susceptibilidad. Las pérdidas que ocasiona la pudrición parda se deben tanto a la pudrición de los frutos en los huertos como durante el transporte y la venta en el mercado. Cuando las infecciones son severas y en ausencia de buenos métodos de control, puede ocurrir que entre el 50 y $75 \%$ de los frutos se pudran en los huertos y que el resto sea infectado antes de que llegue al mercado (Gabilondo et al., 2002; Mitidieri, 2003).

\section{MATERIALES Y MÉTODOS}

\section{Material vegetal}

La recolección del material vegetal se llevó a cabo durante los meses de septiembre de 2017 y marzo de 2018 con sus correspondientes datos: número de registro, fecha, datos geográficos (GPS), condiciones climáticas, tipo de suelo y número de ejemplares recogidos. El material recolectado fue identificado y determinado con la ayuda de lupa estereoscópica y claves dicotómicas. Un ejemplar de las especies $D$. ambrosioides, B. frenguellii, B. salicifolia y L. turbinata fueron depositados en el Herbario "Arturo Ragonese" de la Facultad de Ciencias Agrarias (FCA-UNL). Un ejemplar de L. alba fue depositado en el Herbario del Museo Provincial de Ciencias Naturales "Florentino Ameghino" (Santa Fe). 


\section{Obtención de los aceites esenciales}

Se trabajó con material fresco recolectado como máximo el día previo a la extracción. Se corroboró que el mismo se encontrara libre de insectos o cualquier otro tipo de contaminante. Las partes aéreas correspondientes de cada especie fueron colocadas en un destilador tipo Clevenger que funciona por arrastre con vapor de agua. Previamente se pesó el material vegetal para calcular el rendimiento del aceite esencial obtenido el cual se expresó en $\mathrm{mL}$ por cada $100 \mathrm{~g}$ de material fresco.

\section{Microorganismos y medios}

A partir de frutas que presentaban los síntomas correspondientes a cada enfermedad fúngica, se obtuvieron cultivos monospóricos que fueron caracterizados morfológicamente y depositados en el Centro de Referencia en Micología (CEREMIC) de la FCByF-UNR, en la micoteca de la Estación Experimental INTA San Pedro (INTA-SP) y en el Laboratorio de Microbiología de la Facultad de Ingeniería Química (LMFIQ-UNL). Los códigos identificatorios de las cepas fueron los siguientes: $B$. cinerea (CEREMIC 100-2018), C. acutatum (CEREMIC 13-2019), R. stolonifer (LMFIQ-317), F. semitectum (CEREMIC 012019) y M. fructicola (INTA-SP345). Las cepas se mantuvieron a una temperatura entre 3 y $5{ }^{\circ} \mathrm{C}$ y cada 15 días se repicaron en placas de Petri previamente esterilizadas, utilizando Agar-Papa-Dextrosa (APD) como medio de cultivo. Los inóculos se obtuvieron a partir de repiques frescos, colocando agua destilada y esterilizada sobre la caja de Petri donde se había desarrollado cada hongo. Se adicionó además una gota de detergente Tween-20 no iónico, para facilitar el desprendimiento de los conidios y se procedió al raspado del micelio fúngico con un ansa bacteriológica estéril. La sus- pensión de conidios obtenida se filtró con ayuda de una jeringa de vidrio con torunda de algodón, previamente esterilizada en estufa a $140{ }^{\circ} \mathrm{C}$, y se colectó en tubos Falcon de $15 \mathrm{~mL}$ para luego conservarlos a una temperatura entre 3 y $5{ }^{\circ} \mathrm{C}$. La determinación de la concentración del inóculo se realizó mediante recuento en cámara de Neubauer siguiendo los protocolos establecidos por el Clinical \& Laboratory Standards Institute (CLSI, 2008).

\section{Ensayos antifúngicos}

Placas de Petri de vidrio de $6 \mathrm{~cm}$ de diámetro se cubrieron con $10 \mathrm{~mL}$ de medio APD previamente fundido. Una vez solidificado el mismo, se colocaron en el centro de la placa, $10 \mu \mathrm{L}$ de inóculo fúngico de concentración entre 104 y 105 Unidades Formadoras de Colonias $/ \mathrm{mL}$ (UFC/mL). Luego de evaporada el agua de la solución de conidios, en el centro de la tapa de la placa de Petri se depositaron $15 \mu \mathrm{L}$ de aceite esencial o agua (experimento control). Dicha cantidad de aceite esencial corresponde a una concentración de 1000 ppm calculada según las dimensiones de las placas de Petri. Las placas así preparadas fueron incubadas de forma invertida, de manera que el aceite esencial que se evaporaba entrara en contacto con el medio de cultivo donde el hongo se estaba desarrollando. Una vez que el micelio de las placas control cubrió por completo la superficie del medio [aproximadamente 7 días excepto para $R$. stolonifer (2 días) y F. semitectum (4 días)] se realizaron las medidas del área del micelio desarrollado en cada placa tratada con cada aceite esencial, a través del escaneo de las mismas para su posterior lectura y análisis con el software Image ${ }^{\circledR}$. Los ensayos se realizaron por triplicado, de manera que se obtuvo un valor promedio de los diámetros de crecimiento fúngico que se expresaron 
en porcentaje respecto al $100 \%$ de crecimiento de los experimentos control.

\section{Análisis cromatográfico}

Los aceites esenciales fueron analizados con un Cromatógrafo gaseoso Agilent modelo 7890B acoplado a un Espectrómetro de Masas Agilent modelo 5977, columna: HP-5MS UI ( $30 \mathrm{~m} \times 0,25 \mathrm{~mm}$ con $0,25 \mu \mathrm{m}$ de film). Las condiciones de corrida fueron las siguientes: Inyector $250{ }^{\circ} \mathrm{C}$; temperatura de la columna: $160{ }^{\circ} \mathrm{C}$ mantenido por 3 min llevado a $5{ }^{\circ} \mathrm{C} / \mathrm{min}$ hasta $30{ }^{\circ} \mathrm{C}$; tiempo de corrida: $31 \mathrm{~min}$. Espectrómetro de Masas: full SCAN: 50-400, volumen de inyección: $1 \mu \mathrm{l}$-Split: 1:20. Para identificar los principales componentes, se compararon los espectros de masas de los picos más abundantes con la base de datos que dispone el equipo [Biblioteca de Espectros de Masas del NIST (The NIST Mass Spectral Search Program for the NIST/EPA/NIH
Mass Spectra Library versión 2.0 build 19 de noviembre de 2000)].

\section{Análisis estadístico}

Las diferencias entre los porcentajes promedio de crecimiento fúngico en presencia de cada muestra de aceite esencial y los controles de crecimiento de cada hongo, se analizaron estadísticamente a través del test de Tukey con intervalos de confianza (IC) del 95\% usando el software GraphPad Prism 5.0 (San Diego, CA, 2010).

\section{RESULTADOS Y DISCUSIÓN}

En la Tabla 1 se muestran los números de Voucher specimen y los rendimientos de los aceites esenciales obtenidos de cada una de las especies evaluadas en este trabajo.

Tabla 1: Número de Voucher specimen y rendimientos ( $\mathrm{mL} / 100 \mathrm{~g}$ de planta fresca) de los aceites esenciales obtenidos de cada especie vegetal estudiada.

Table 1: Voucher specimen number and essential oil yields ( $\mathrm{mL} / 100 \mathrm{~g}$ of fresh material) of each plant species under study.

\begin{tabular}{|l|l|l|c|}
\hline \multicolumn{1}{|c|}{ Especie vegetal } & \multicolumn{1}{|c|}{$\mathrm{N}^{0}$ Voucher specimen } & \multicolumn{1}{|c|}{ Lugar de colecta } & Rendimiento \\
\hline D. ambrosioides & MD40 UNL & $\begin{array}{l}\text { Reserva escuela granja, Esperanza } \\
\text { (Sta. Fe) }\end{array}$ & $0,40 \%$ \\
\hline & & $\begin{array}{l}\text { Ruta 70 y América, Esperanza (Sta. } \\
\text { Fe) }\end{array}$ & $0,23 \%$ \\
\cline { 3 - 4 } B. frenguellii & MD49 UNL & $\begin{array}{l}\text { Ruta 70 y América, Esperanza (Sta. } \\
\text { Fe) }\end{array}$ & $0,42 \%$ \\
\hline B. salicifolia & MD48 UNL & Depto. La Capital (Sta. Fe) & $0,39 \%$ \\
\hline & & & \\
\hline L. alba & Olivella, Getar y & $\begin{array}{l}\text { Reserva Escuela Granja, } \\
\text { Esperanza (Sta. Fe) }\end{array}$ & $0,45 \%$ \\
\hline L. turbinata & Gutiérrez 05(FHUC) & MD56 UNL &
\end{tabular}




\section{I. Stegmayer et al.}

Como se puede observar en la Tabla 1, los rendimientos de los aceites esenciales fueron menores al 0,5\% siendo D. ambrosioides la especie que mayor cantidad de aceite esencial destiló y $\mathrm{B}$. frenguellii la que produjo menor cantidad. Estos resultados están de acuerdo con lo ampliamente conocido respecto a la baja proporción de aceites esenciales que destilan las plantas (Terblanché, 1996).
En la Figura 1 se muestran las áreas de crecimiento promedio de cada hongo ( $B$. cinerea, C. acutatum, $R$. stolonifer, F. semitectum y $M$. fructicola) ensayado frente a 1000 ppm de los aceites esenciales de las plantas evaluadas en las condiciones de trabajo. Las áreas de crecimiento que presentaron diferencias significativas entre sí o con el experimento control, se marcaron con letras diferentes.

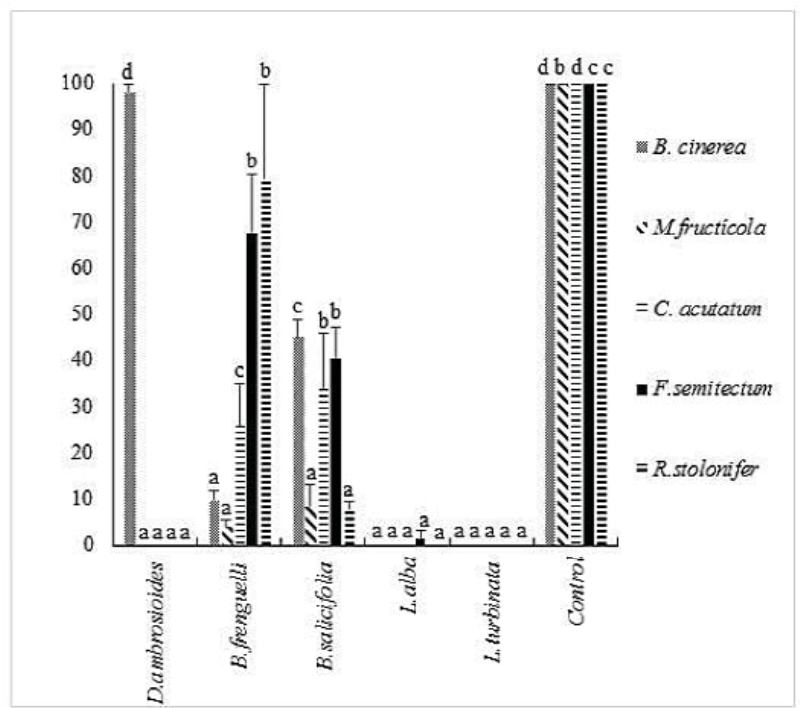

Figura 1: Áreas de crecimiento promedio de los hongos B. cinerea, M. fructicola, C. acutatum, $F$. semitectum y $R$. stolonifer correspondientes a los tratamientos con los aceites esenciales obtenidos de las plantas nativas D. ambrosioides, B. frenguellii, B. salicifolia, L. alba, L. turbinata y control. Letras iguales no presentan diferencias significativas ( $p \geq 0,05)$.

Figure 1: Average growth areas of B. cinerea, M. fructicola, C. acutatum, F. semitectum and $R$. stolonifer corresponding to treatments with essential oils obtained from native plants $D$. ambrosioides, B. frenguellii, B. salicifolia, L. alba, L. turbinata and control. Same letters means no significant differences ( $p \geq 0.05$ ). 
Como se puede observar en la Figura 1 , el aceite esencial de D. ambrosioides no permitió el desarrollo de C. acutatum, $R$. stolonifer, F. semitectum y $M$. fructicola inhibiéndolos al $100 \%$, pero distinto fue su comportamiento frente a $B$. cinerea donde se observó un $95 \%$ de crecimiento, no presentando diferencias significativas respecto al control. Esto indicaría un comportamiento antifúngico selectivo frente a algunos tipos de patógenos de frutas y se sumarían al panel de hongos evaluados por Jardim et al. (2008). El aceite esencial de $B$. frenguellii resultó activo contra $B$. cinerea y $M$. fructicola desarrollando apenas áreas de crecimientos de 10 y $4 \%$ respectivamente que presentaron diferencias significativas respecto al control, mientras que fue moderadamente activo contra $C$. acutatum $(25 \%$ de área de crecimiento respecto al control) y muy poco activo frente a $F$. semitectum y $R$. stolonifer que presentaron áreas de crecimiento del 70 y $80 \%$ respectivamente, aun diferenciándose con el control. Estos resultados amplían la evaluación de Feresín et al. (2001) quienes demostraron actividad antifúngica de otra especie de Baccharis contra dermatofitos humanos pero nunca se había explorado su acción fungicida contra fitopatógenos. Respecto al aceite esencial de $B$. salicifolia, demostró una fuerte actividad contra M. fructicola y R. stolonifer con áreas de crecimiento menores al $10 \%$ comparadas con el control y una actividad moderada frente a los restantes patógenos evaluados, con áreas de crecimiento entre 30 y $50 \%$ respecto al control, del que se diferenciaron significativamente de todas maneras. Estos resultados profundizan la exploración de Ávila-Sosa et al. (2011) quienes mencionaron una inhibición del 88\% del fitopatógeno C. gloeosporioides luego de la exposición a un extracto clorofórmico obtenido de esta especie vegetal. Finalmente, los aceites esenciales obtenidos de ambas especies de Lippia resultaron ser los más activos, dado que impidieron el desarrollo de todos los hongos evaluados en las condiciones del ensayo excepto un ínfimo crecimiento (2\%) de F. semitectum tratado con L. alba. Esto nos indicaría que estamos frente a dos productos antifúngicos muy potentes y de amplio espectro de acción, resultados consistentes con los de Shukla et al. (2009), Rao et al. (2000) y de Oliveira et al. (2019) que demostraron la fuerte inhibición de varios patógenos con el aceite esencial de $L$. alba. Respecto a $L$. turbinata, hasta donde conocemos, es la primera vez que se informa su actividad antifúngica.

En la Tabla 2 se presenta una breve descripción de los cinco compuestos mayoritarios y sus porcentajes presentes en cada uno de los aceites esenciales estudiados. Estos datos se corresponden en mayor o menor medida con las descripciones de la literatura sobre la composición de estos aceites (Alonso y Desmarchelier, 2005; Carrizo-Flores et al., 2009; Feresín et al., 2001; Oliveira et al., 2006; Terblanche y Kornelius, 1996), teniendo en cuenta la amplia variabilidad química que caracteriza a los aceites volátiles. Como se puede observar, los compuestos mayoritarios son mono o sesquiterpenos provenientes de la vía del mevalonato (carvacrol, eucaliptol, timol, limoneno, careno, linalol, carvona, entre otros) pero también en algunos casos se observan derivados del ácido shikímico como el metileugenol y algunos derivados oxigenados también se hallan presentes sobre todo en $B$. salicifolia. 
M. I. Stegmayer et al.

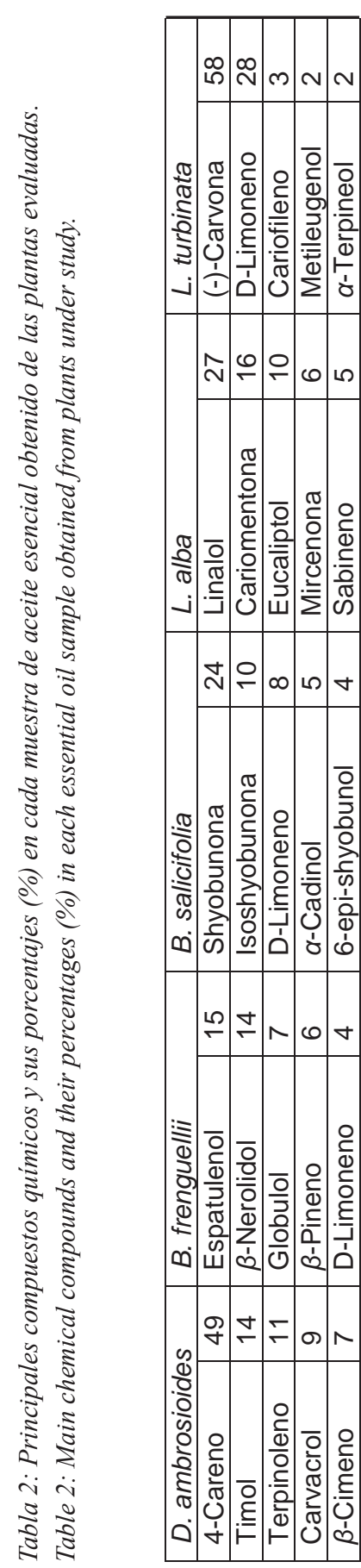




\section{CONCLUSIONES}

Los cinco aceites esenciales provenientes de las plantas nativas evaluadas en este trabajo con el fin de encontrar algún potencial fungicida para el control de los fitopatógenos B. cinerea, C. acutatum, $R$. stolonifer, F. semitectum y M. fructicola, resultaron de alta eficacia en algunos casos y moderada en otros. A las concentraciones evaluadas, los aceites provenientes de las especies de Lippia resultaron activos frente a todos los hongos del panel inhibiendo su desarrollo completamente, por lo que continuaremos los ensayos utilizando menores dosis de modo de detectar la concentración fungicida mínima y así poder probar estos productos en frutas inoculadas con estos patógenos. Nuestros resultados destacan la gran riqueza que presenta la flora nativa argentina como fuente de potenciales antifúngicos naturales.

\section{AGRADECIMIENTOS}

Los autores agradecen al Consejo Nacional de Investigaciones Científicas y Técnicas (CONICET), a la Agencia Nacional de Promoción Científica y Tecnológica (ANPCyT) y a la Universidad Nacional del Litoral (UNL) por los soportes financieros: PIP N ${ }^{\circ}$ 2015-0524, PICT N 2015-2259, $\mathrm{CAI}+\mathrm{D}$ 2016-50020150100034LI. MIS, LNF, NHA y LO también agradecen al CONICET por sus becas doctorales.

\section{BIBLIOGRAFÍA}

Alonso J, Desmarchelier C. 2005. Plantas medicinales autóctonas de la Argentina. Lola. Buenos Aires. 663 pp.

Avila-Sosa R, Gastélum-Reynoso G, García-Juárez M, Meneses-Sánchez M, Navarro-Cruz AR, Dávila-Márquez RM. 2011. Evaluation of different Mexican plant extracts to control anthracnose. Food and Bioprocess Tech. 4(4):655-659.

Bolda M, Daugovish O, Koike S. 2017. Ficha $\mathrm{n}^{\circ} 14$ Antracnosis de la fresa. Comisión de la Fresa de California; [accedido el 15 diciembre de 2019]. https://ucanr.edu/blogs/fresamora/blogfiles/47936.pdf.

Cámara de Sanidad y Fertilizantes. 2017-2019. República Argentina-CASAFE. Guía de productos fitosanitarios para la República Argentina. $18^{\mathrm{a}}$ Ed. Buenos Aires: CASAFE. 1368 pp.

Campos-Navarro R, Scarpa GF. 2013. The cultural-bound disease "empacho" in Argentina. A comprehensive botanico and ethnopharmacological review. J Ethnopharmacol. 148(2): 349360 .

Carrizo Flores R, Ponzi M, Ardanaz C, Tonn CE, Donadel OJ. 2009. Chemical composition of essential oil of Baccharis salicifolia (Ruiz \& Pavon) Pers. and antibacterial activity. J Chil Chem Soc. 54(4):475-476.

Clinical and Laboratory Standars Institute (CLSI). 2008. Reference method for broth dilution antifungal susceptibility testing of filamentous fungi. En: CLSI document M-38 A2. $2^{\circ}$ Ed. Pennsylvania: Wayne (Ed.). Pp. 1-35.

De Oliveira Arruda RD, Victório CP, Boaretto AG, Carollo CA, Da Silva Farias C, Marchetti CR, Silva DB. 2019. Essential oil composition, antifungal activity and leaf anatomy of Lippia alba (Verbenaceae) from Brazilian Chaco. J Med Plant Res. 13(4):79-88. 
Di Liberto M, Stegmayer MI, Svetaz L, Derita M. 2019. Evaluation of argentinean medicinal plants and isolation of their bioactive compounds as an alternative for the control of postharvest fruits phytopathogenic fungi. Br J Pharmacognosy. 29:686-689.

Di Stasi LC, Hiruma CA, Guimarães EM, Santos CD. 1994. Medicinal plants popularly used in Brazilian Amazon. Fitoterapia. 65(6):529-540.

Eyssartier C, Ladio AH, Lozada M. 2009. Uso de plantas medicinales cultivadas en una comunidad semi-rural de la estepa patagónica. BLACPMA 8(2):77-85.

Feresín GE, Tapia A, López SN, Zacchino SA. 2001. Antimicrobial activity of plants used in traditional medicine of San Juan province, Argentine. J Ethnopharmacol. 78(1):103-107.

Freire SE, Urtubey E, Giuliano DA. 2007. Caracteres epidérmicos de las especies del género Baccharis (Asteraceae) usadas en la medicina popular. Caldasia. 29(1):23-39.

Gabilondo J, Bordoli RJ, Mitidieri MS. 2002. Comportamiento frente a carbendazim de cepas de Monilia fructicola provenientes de lotes de producción integrada de duraznero. Documento presentado en: Libro de resúmenes de IX Congreso Argentino Ciencia y Tecnología Alimentos. Asociación Argentina de Tecnólogos Alimentarios. Pp. 7-9.

Giusti L. 1997. Chenopodiaceae. Museo Botánico, IMBIV, Programa Proflora (Conicet). Disponible en: http://www.floraargentina.edu. ar/wp-content/uploads/2019/01/40-CHENOPODIACEAE.pdf.

González BA. 2017. Antracnosis o Mancha negra en frutilla. [Accedido el 11 diciembre de 2019]. Disponible en: http://www.patologiavegetal.unlu.edu.ar/?q=node/69\#ANTRACNOSIS.
Hernández NE, Tereschuk ML, Abdala LR. 2000. Antimicrobial activity of flavonoids in medicinal plants from Tafi del Valle (Tucuman, Argentina). J Ethnopharmacol. 73(1):317-322.

Jardim CM, Jham GN, Dhingra OD, Freire MM. 2008. Composition and antifungal activity of the essential oil of the Brazilian Chenopodium ambrosioides L. J Chem Ecol. 34(9):1213-1218.

Kim Y, Lee C, Kim H, Lee H. 2004. Anthraquinones isolated from Cassia tora (Leguminosae) seed and antifungical property against phytopatogenic fungi. J Agric Food Chem. 52:6096-6100.

Martinez MJ, Bessa AL, Benito, PB. 2005. Biologically active substances from the genus Baccharis L. (Compositae). En: Studies in Natural Products Chemistry. Elsevier. Pp. 703-759.

Mitidieri M. 2003. Enfermedades del duraznero. INTA. Disponible en: http://anterior.inta.gov. ar/sanpedro/info/doc/prv/mm_010.htm.

Munitz M. 2015. Arándanos: micoflora contaminante, micotoxinas, residuos de fungicidas y cinéticas de degradación. Tesis Doctoral. Facultad de Cs Exactas y Naturales. Universidad de Buenos Aires.

Navone GT, Zonta M, Gamboa M. 2014. Fitoterapia Mbyá-Guaraní en el control de las parasitosis intestinales: Un estudio exploratorio con Chenopodium ambrosioides L. var anthelminticum en cinco comunidades de Misiones, Argentina. Polibotánica. 37:135151.

Oliveira DR, Leitao GG, Santos SS, Bizzo HR, Lopes D, Alviano CS, Leitao SG. 2006. Ethnopharmacological study of two Lippia species from Oriximiná, Brazil J Ethnopharmacol. 108(1):103-108. 
Passone MA, Etcheverry M. 2014. Antifungal impact of volatile fractions of Peumus boldus and Lippia turbinata on Aspergillus section Flavi and residual levels of these oils in irradiated peanut. Int J Food Microbiol. 168:17-23.

Pergomet JL, Di Liberto MG, Derita MG, Bracca ABJ, Kaufman TS. 2018. Activity of the pterophyllins 2 and 4 against postharvest fruit pathogenic fungi. Comparison with a synthetic analog and related intermediates. Fitoterapia. 125:98-105.

Prasad C, Shukla R, Kumar A, Dubey N. 2010. In vitro and in vivo antifungal activity of essential oils of Cymbopogon martini and Chenopodium ambrosioides and their synergism against dermatophytes. Mycoses. 53(2):123-129.

Rao G, Singh M, Singh P, Singh S, Catalan C, Kapoor I, Singh G. 2000. Studies on chemical constituents and antifungal activity of leaf essential oil of Lippia alba (Mill). Indian J Chem Technol. 7(6):332-335.

Salem EA, Youssef K, Sanzani SM. 2016. Evaluation of alternative means to control postharvest Rhizopus rot of peaches. Sci Hortic. 198: 86-90.

Scarpa GF. 2004. Medicinal plants used by the Criollos of Northwestern Argentine Chaco. J Ethnopharmacol. 91(1):115-135.

Seta S, Gonzales M, Lori G. 2004. First report of walnut canker caused by Fusarium incarnatum in Argentina. Plant Pathol. 53:248.

Shukla R, Kumar A, Singh P, Dubey NK. 2009. Efficacy of Lippia alba (Mill.) NE Brown essential oil and its monoterpene aldehyde constituents against fungi isolated from some edible legume seeds and aflatoxin $\mathrm{B} 1$ production. Int J Food Microbiol. 135(2):165-170.
Singh H, Batish D, Kohlil R. 2003. Allelopathic interactions and allelochemicals: new possibilities for sustaintable weed management. Crit Rev Plant Sci. 22:239-311.

Terblanché FC, Kornelius G. 1996. Essential oil constituents of the genus Lippia (Verbenaceae)-a literature review. J Essent Oil Res. 8(5):471-485.

You J, Zhang J, Wu M, Chen W, Li G. 2016. Multiple criteria-based screening of Trichoderma isolates for biological control of Botrytis cinerea on tomato. Biological Control. 101: 31-38. 